\title{
PREDICCIÓN DEL ACRETISMO PLACENTARIO CON ULTRASONIDO DOPPLER EN EL HOSPITAL UNIVERSITARIO DE NEIVA, COLOMBIA. ESTUDIO DE COHORTE
}

\section{Predicting placenta accreta with Doppler ultrasound at the Neiva teaching hospital, Collombia. A cohort study}

Fidel Ernesto Ferreira-Narváez, M.D.*, Mónica Angulo-Carvallo**

Recibido: noviembre 27/06 - Revisado: agosto 13/07 - Aceptado: octubre 30/07

\section{RESUMEN}

Objetivo: evaluar los hallazgos ecográficos, el resultado materno y perinatal de las pacientes con y sin acretrismo placentario a quienes se les realizó ecografía y doppler color antenatal.

Métodos: se realizó un estudio de cohorte en pacientes con riesgo de acretismo placentario describiendo los resultados de la ecografía doppler y comparándolos con los hallazgos operatorios en el parto, la evolución del mismo, la necesidad de histerectomía y el resultado perinatal.

Resultados: un total de 51 pacientes con riesgo de acretismo fueron evaluadas mediante ecografía y doppler color. En cinco pacientes se confirmó alta probabilidady en 46 pacientes baja probabilidad de acretismo placentario. Comparando el resultado en términos de presencia o ausencia de acretismo, la tasa de histerectomía fue 60\% en el grupo de alta probabilidad y $2 \%$ en el grupo de baja probabilidad. Las transfusiones sanguíneas fueron necesarias en un $40 \%$ de las pacientes de alta probabilidad y $2 \%$ en el grupo de baja probabilidad.

\footnotetext{
* Perinatólogo. Profesor asistente. Departamento de Ginecología y Obstetricia. Universidad Surcolombiana. Coordinador Unidad de Alto Riesgo Obstétrico. Hospital Universitario Hernando Moncaleano Perdomo, Neiva, Colombia.

**Residente Ginecología y Obstetricia. Universidad Surcolombiana. Unidad de Alto Riesgo Obstétrico, Hospital Universitario Hernando Moncaleano Perdomo, Neiva, Colombia.

Correspondencia: carrera 38A \# 8-15, Apto. 403, Ipanema, Neiva, Colombia Correo electrónico: angulocarvallomonica@hotmail.com
}

Conclusiones: las complicaciones maternas y neonatales son más frecuentes en pacientes con alta probabilidad de acretismo placentario. El diagnóstico antenatal de acretismo placentario es fundamental en la identificación de pacientes en riesgo de complicaciones y necesidad de cesárea seguida de histerectomía.

Palabras clave: acretismo placentario, placenta previa, ultrasonografía doppler, histerectomía, parto por cesárea.

\section{SUMMARY}

Objective: evaluating ultrasonographic findings and maternal and perinatal outcomes for patients with and without accretism who were submitted to antenatal ultrasonographies and Doppler ultrasound.

Methods: a cohort of high-risk women was studied for accretism. Foetal Doppler results were compared with outcomes after surgery, the need for hysterectomy, perinatal outcome and clinical evolution following surgery.

Results: a total of 51 patients having a high risk of accretism were evaluated. High-probability for accretism was confirmed in 5 patients, whereas lowprobability was found for 46 patients. Comparing the results in terms of the presence of accretism, 
there was a $60 \%$ hysterectomy rate for the highprobability group whereas it was only $2 \%$ in the low-probability group. Blood transfusions were necessary in $40 \%$ of patients having high-risk of accretism, whereas it was only $2 \%$ in the low-probability grup.

Conclusions: maternal and neonatal complications are more frequent in patients having a high probability of accretism. Prenatal diagnosis of placenta accreta is essential in detecting patients at risk of maternal complications and determining the need for hysterectomy following a caesarean section.

Key words: placenta accreta, placenta previa, hysterectomy, Doppler ultrasonography.

\section{INTRODUCCIÓN}

Bajo el término de placenta acreta se define la invasión de las capas musculares del útero por parte de la placenta, en este caso se denomina placenta increta, y cuando se presenta invasión de la serosa u órganos vecinos (ej.: vejiga), hablamos de placenta percreta. ${ }^{1} \mathrm{Su}$ frecuencia se ha estimado en 1-3\% para nulíparas y se incrementa con la presencia de cicatrices uterinas por cesárea o miomectomía. ${ }^{1,2} \mathrm{La}$ incidencia de acretismo placentario es actualmente 10 veces mayor que hace tres décadas, en los Estados Unidos se presenta en el 9,3\% de las mujeres con placenta previa; tendencia debida al aumento de la tasa de cesáreas estimada en 29,1\% de los nacimientos para el año 2004..$^{2-4}$

Esta condición tiene una importante morbimortalidad asociada; informándose una mortalidad materna de $7 \%$ relacionada con hemorragia masiva en las pacientes afectadas con placenta acreta. ${ }^{5-7}$

Se ha estimado un riesgo de placenta acreta del 10 a 25\% en mujeres con una cesárea previa y mayor a $50 \%$ con antecedente de dos o más cesáreas. ${ }^{3,8}$ Otros factores que incrementan el riesgo de acretismo placentario son la edad materna mayor de 35 años, la alta paridad, el consumo de cigarrillo, los miomas submucosos y el síndrome de Asherman. ${ }^{1-3}$

Para las pacientes con estos factores de riesgo y placenta de localización anterior o previa es necesario descartar o confirmar el diagnóstico de acretismo en el período antenatal, ya que es importante hacer un manejo programado y multidisciplinario, ${ }^{7}$ con el objetivo de disminuir la morbilidad debida a transfusión masiva, infección, lesión urológica y formación de fístulas así como la mortalidad materna y neonatal. , $^{3,910}$ Por lo tanto, los métodos para lograr un diagnóstico temprano de acretismo placentario son determinantes en el manejo adecuado de esta patología. ${ }^{4-6}$

La ultrasonografía es útil en la búsqueda de placenta acreta, y requiere una valoración cuidadosa y diligente de la interfase uteroplacentaria. La ausencia o adelgazamiento (menor de $1 \mathrm{~mm}$ ) de la zona miometral hipoecoica normal en el segmento uterino anterior es sugestivo de una adhesión anormal de la placenta. ${ }^{11,12}$ La sensibilidad (93\%), especificidad (79\%), y el valor predictivo positivo (78\%) de este criterio sonográfico son aceptables según pequeñas series, ${ }^{13-16}$ pero combinado con otros criterios ecográficos se puede establecer el diagnóstico de placenta acreta confiablemente. La presencia de la zona miometrial hipoecoica normal es consistente con una baja probabilidad $(<10 \%)$ de placenta acreta. ${ }^{15,16}$ La presencia de espacios lacunares vasculares en el parénquima placentario se le ha llamado "apariencia en queso Suizo", se piensa que este hallazgo es un factor de riesgo para placenta acreta, aun en ausencia de otros hallazgos sospechosos. ${ }^{15-17} \mathrm{El}$ adelgazamiento, irregularidad o disrupción focal de la interfase hiperecoica entre la pared vesical y el útero, es muy sospechosa de invasión de la vejiga. ${ }^{18}$

El doppler color puede incrementar el nivel de confiabilidad en la identificación de la zona miometrial. ${ }^{17}$ Otras ventajas del doppler color son la mayor especificidad en el diagnóstico de placenta acreta y una mejor valoración de la profundidad de la invasión miometrial o de la serosa. Cuando se pueden identificar los vasos prominentes extendiéndose desde la base de la placenta al miometrio o la pared vesical, se puede sospechar el diagnóstico de placenta acreta. ${ }^{6,10.13,17}$

La imagen de resonancia magnética (IRM) puede ser muy útil, particularmente para evaluar la inva- 
sión vesical. ${ }^{17,19}$ Otra ventaja de la IRM es la habilidad para visualizar la invasión de la pared posterior y lateral del útero en esos casos en que la placenta se implantó posteriormente. La IRM se debe usar en los casos en que el ultrasonido no pueda descartar la presencia de placenta acreta. ${ }^{10,17,18}$

En nuestro medio el uso de la ecografía doppler es la herramienta diagnóstica de primera línea, debido a su importante sensibilidad, mayor accesibilidad y menor costo.

El objetivo de este estudio es determinar la capacidad predictora de la ultrasonografía en el diagnóstico antenatal de acretismo placentario.

\section{MATERIALES Y MÉTODOS}

Diseño: estudio de cohorte de mujeres con alto riesgo de acretismo placentario en quienes se practicó utrasonografía doppler entre las 20 y 40 semanas y se determinó el resultado materno y perinatal.

En este estudio no se realizó ninguna intervención de tipo experimental, las pacientes llenaron el formato de consentimiento informado de atención hospitalaria, aplicándose en cada caso el protocolo de manejo establecido según las guías de la institución.

Población y lugar: mujeres con alto riesgo de acretismo placentario atendidas entre agosto del 2005 y septiembre del 2006, en el Hospital Universitario Hernando Moncaleano Perdomo de la ciudad de Neiva, Colombia. Hospital de alta complejidad que atiende pacientes de bajos recursos afiliados a la seguridad social contributiva, subsidiada y quienes carecen de ella.

Criterios de inclusión: mujeres con alto riesgo de acretismo placentario: antecedente de cesárea(s) con placenta de localización anterior y pacientes con placenta previa.

Criterios de exclusión: pacientes que no aceptaran la realización de la prueba diagnóstica y/o falta de datos en la historia clínica.

La valoración sonográfica fue llevada a cabo con un equipo de ultrasonido Toshiba Nemio 4D (Toshiba medical systems corp. 2001-2004).
Las pacientes fueron clasificadas como de alta o baja probabilidad de acretismo placentario según los hallazgos descritos.

El seguimiento de la evolución de las pacientes hasta el parto, el curso del parto y complicaciones subsecuentes, así como el resultado perinatal, fueron analizados a través de la revisión de historias clínicas. La información fue recogida mediante la aplicación de un formulario.

Las variables analizadas fueron:

Variables predictoras: edad materna, edad gestacional, paridad, ubicación de la placenta, antecedente y número de cesáreas previas.

Se definió ultrasonografía de alta probabilidad de acretismo placentario a aquellas que presentaron pérdida de la interfase placenta-miometrio, ${ }^{13,18}$ invasión vascular al miometrio vista por doppler color y power angio, presencia de lagunas placentarias y adelgazamiento de la interfase entre la pared vesical y la placenta. ${ }^{15-17}$ (Figura 1).

Se definió ultrasonografía de baja probabilidad a aquellas en donde no se encontraron los hallazgos previamente mencionados y se visualizó adecuadamente la interfase placentamiometrio.

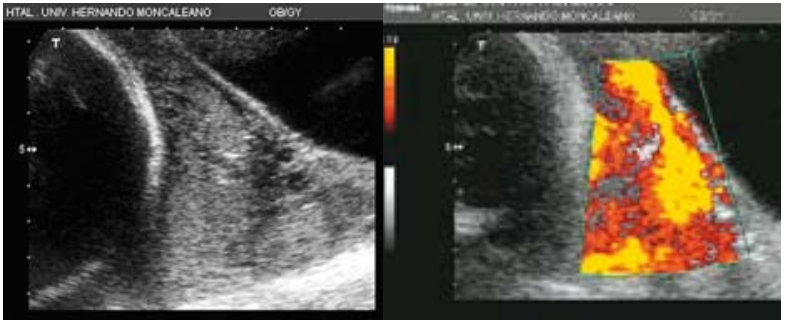

Figura 1. Fidel Ernesto Ferreira. Unidad de Alto Riesgo Obstétrico. Hospital Universitario Hernando Moncaleano Perdomo. Neiva, Colombia. Imagen por ecografía doppler con alta probabilidad de acretismo placentario. HUN, 2005-2006.

Variables resultado: acretismo al momento del parto, definido por la presencia de infiltración placentaria en el miometrio y la serosa uterina visualizada macrosocópicamente y dificultad para la extracción manual de la placenta. ${ }^{20}$

Se definió como pacientes sin acretismo al momento del parto, aquellas que presentaron fácil 
desprendimiento de la placenta sin los hallazgos mencionados previamente.

Tipo de parto (por cesárea o vaginal), morbilidad neonatal (Apgar del recién nacido, prematurez, peso al nacer), morbilidad materna (trasfusión, ingreso a UCI, histerectomía) y muerte.

Los datos fueron analizados con Epi-info versión 3.3.2. Las características basales de las pacientes se describen por medio de la media, varianza, desviación típica, mediana y moda.

Para la prueba diagnóstica se determinó la sensibilidad y especificidad en la predicción de acretismo al momento del parto.

\section{RESULTADOS}

Un total de 51 pacientes con riesgo de acretismo fueron evaluadas mediante ecografía y doppler color. La sensibilidad de la ecografía doppler color para acretismo placentario al momento del parto fue 100\% y la especificidad 96\%.

De las 51 pacientes, cinco tenían alta probabilidad y 46 baja probabilidad para acretismo placentario.

En el grupo de cinco pacientes con alta probabilidad de acretismo, 4 de 5 tenían antecedente de cesárea y todas presentaban placenta previa oclusiva total, en este grupo se confirmó el diagnóstico de acretismo placentario de forma intraoperatoria en 3 de 5 pacientes (figura 2). La edad gestacional al parto fue en promedio 33 semanas; la vía del parto en todos los cinco casos fue la cesárea, dos de las cinco pacientes requirieron transfusión de hemoderivados, dos de las cinco pacientes manejo en unidad de cuidados intensivos, a tres de cinco pacientes se les realizó histerectomía, y se presentó una muerte materna.

En cuanto al resultado neonatal el peso al nacer en este grupo en promedio fue 2.295 gramos. Las carateristicas basales de los dos grupos se presentan en la tabla 1.

En las 46 mujeres del grupo de baja probabilidad ninguna presentó hallazgos intraoperatorios de acretismo placentario. Cuarenta y cuatro de las cuarenta y seis pacientes tenían antecedente de parto por

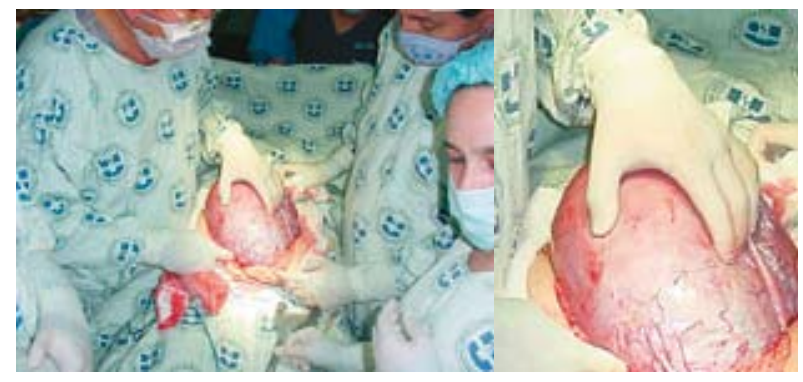

Figura 2. Hallazgos intraoperatorios de acretismo placentario, HUN 2005-2006.

cesárea. La tasa de placenta previa total fue menor (2 de 46). Ninguna requirió ingreso a la unidad de cuidados intensivos, solo en un caso se realizó histerectomía con necesidad de trasfusión debido a atonía uterina poscesárea, descartándose en el espécimen de patología el acretismo y no hubo mortalidad en este grupo. Dentro de los resultados perinatales, la edad gestacional al parto en promedio fue de 38,3 semanas, la vía del parto más frecuente fue la cesárea (36 de 46) y el peso fetal en promedio fue de 3.180 gramos. Tablas 1-3

\section{DISCUSIÓN}

En el grupo de 51 pacientes con riesgo de acretismo placentario evaluadas en este estudio, se determinó mediante ecografía y doppler color que cinco pacientes tenían alta probabilidad y 46 baja probabilidad de acretismo placentario. La sensibilidad y especificidad de la ecografía doppler color para el hallazgo operatorio de acretismo placentario al momento del parto fue $100 \%$ y $96 \%$ respectivamente. Carri y cols. han informado una sensibilidad y especificidad de $77 \%$ y $96 \%$ en su orden. ${ }^{3}$ El hallazgo de alta probabilidad de acretismo placentario mediante ecografía y doppler color se relacionó con una mayor morbilidad materna, menor edad gestacional al parto y menor peso al nacimiento, confirmando de esta manera los factores de riesgo en este grupo de pacientes. El estándar de oro utilizado para determinar la sensibilidad y especificidad de la ecografía en nuestra serie fue el hallazgo operatorio y no el resultado de anatomía patológica, debido a que en nuestra institución no se realiza de forma rutinaria estudio de patología a todas las pacientes; sin embargo, a futuro esperamos 
Tabla 1. Características de las maternas con factores de riesgo para acretismo placentario. Hospital

Universitario de Neiva (HUN), 2005-2006.

\begin{tabular}{|l|c|c|}
\hline \multicolumn{1}{c|}{ Características } & $\begin{array}{c}\text { Baja probabilidad } \\
\text { (n: 46) }\end{array}$ & $\begin{array}{c}\text { Alta probabilidad } \\
\text { (n: 5) }\end{array}$ \\
\hline $\begin{array}{l}\text { Promedio edad } \\
\text { (años) }\end{array}$ & 28 & 38 \\
\hline $\begin{array}{l}\text { Promedio } \\
\text { gestaciones }\end{array}$ & 3 & 6 \\
\hline $\begin{array}{l}\text { Promedio de } \\
\text { cesáreas previas }\end{array}$ & 1 & 2 \\
\hline $\begin{array}{l}\text { Localización de la } \\
\text { placenta }\end{array}$ & $2(4,3 \%)$ \\
\hline $\begin{array}{l}\text { Corporal anterior } \\
\text { Fúndica y corporal } \\
\text { anterior }\end{array}$ & $38(82,6 \%)$ & 0 \\
\hline $\begin{array}{l}\text { Corporal } \\
\text { antero-lateral }\end{array}$ & $2(6,5 \%)$ & 0 \\
\hline $\begin{array}{l}\text { Previa oclusiva } \\
\text { total }\end{array}$ & $1(2,2 \%)$ & $5(100 \%)$ \\
\hline \begin{tabular}{l} 
Previa posterior \\
\hline
\end{tabular} & & 0 \\
\hline
\end{tabular}

Tabla 2. Resultados de las maternas con factores de riesgo para acretismo placentario. HUN, 2005-2006.

\begin{tabular}{c|c|c|}
$\begin{array}{c}\text { Resultados } \\
\text { maternos }\end{array}$ & $\begin{array}{c}\text { Baja probabilidad } \\
(\mathrm{n}: 46)\end{array}$ & $\begin{array}{c}\text { Alta probabilidad } \\
(\mathrm{n}: 5)\end{array}$ \\
\hline
\end{tabular}

Hallazgo operatorio de acretismo

0

3 placentario

\begin{tabular}{l|c|c|}
$\begin{array}{l}\text { Edad gestacional al } \\
\text { parto } \\
\text { Vía del parto }\end{array}$ & 38,3 semanas & 33 semanas \\
\hline Parto cesárea & 36 & 5 \\
\hline Parto vaginal & 10 & 0 \\
\hline Trasfusión & 1 & 2 \\
\hline Ingreso a UCI & 0 & 2 \\
\hline Muerte & 0 & 1 \\
\hline Histerectomía & 1 & 3 \\
\hline
\end{tabular}

Tabla 3. Resultados neonatales de pacientes con factores de riesgo para acretismo placentario. HUN, 2005-2006

\begin{tabular}{l|c|c|} 
Resultado neonatal & $\begin{array}{c}\text { Baja probabilidad } \\
\text { (n: 46) }\end{array}$ & $\begin{array}{c}\text { Alta probabilidad } \\
\text { (n: 5) }\end{array}$ \\
\hline $\begin{array}{l}\text { Edad gestacional al } \\
\text { parto }\end{array}$ & 38,3 semanas & 33 semanas \\
\hline Peso al nacer & $3.180 \mathrm{~g}$ & $2.295 \mathrm{~g}$ \\
\hline Apgar & 9 y 10 & 7 y 8 \\
\hline
\end{tabular}

realizar estudios prospectivos que nos permitan mejorar estas debilidades metodológicas.

\section{CONCLUSIONES}

Las pacientes con acretismo placentario tienen mayores tasas de morbilidad y riesgo de mortalidad, por lo que es necesario tener un diagnóstico antenatal preciso para así llevar a cabo un manejo racional y programado tendiente a disminuir complicaciones graves.

La ecografía doppler es una herramienta fundamental y altamente exacta para determinar acretismo placentario, siendo el examen de primera línea en la búsqueda de dicha patología. Las pruebas diagnósticas tendientes a la detección antenatal de acretismo placentario son útiles en todos los casos de placenta previa, así como en las pacientes con antecedente de cesárea y placenta sobre la pared anterior y el segmento uterino.

\section{REFERENCIAS}

1. Oyelese Y, Smulian JC. Placenta previa, placenta accreta, and vasa previa. Obstet Gynecol 2006;107:927-41.

2. Miller DA, Chollet JA, Goodwin TM. Clinical risk factors for placenta previa-placenta accreta. Am J Obstet Gynecol 1997;177:210-4.

3. Warshak CR, Eskander R, Hull AD, Scioscia AL, Mattrey RF, Benirschke K, et al. Accuracy of ultrasonography and magnetic resonance imaging in the diagnosis of placenta accreta. Obstet Gynecol 2006;108:573-81.

4. Clark SL, Koonings PP, Phelan JP. Placenta previa/accreta and prior cesarean section. Obstet Gynecol 1985;66:89-92.

5. Chou MM, Tseng JJ, Ho ES, Hwang JI. Threedimensional color power Doppler imaging in the assessment of uteroplacental neovascularization in placenta previa increta/percreta. Am J Obstet Gynecol 2001;185:1257-60.

6. Comstock CH, Love JJ Jr, Bronsteen RA, Lee W, Vettraino IM, Huang RR, et al. Sonographic detection of placenta accreta in the second and third trimesters of pregnancy. Am J Obstet Gynecol 2004;190,1135-40.

7. O'Brien JM, Barton JR, Donaldson ES. The management of placenta percreta: conservative and operative 
strategies. Am J Obstet Gynecol 1996;175:1632-8.

8. Clark SL. Placenta previa and abruptio placentae. En: Creasy RK, Resnik R (eds.). Maternal-fetal medicine: principles and practice. 5th ed. Philadelphia: Saunders; 2004. p.707-22.

9. Yang JI, Lim YK, Kim HS, Chang KH, Lee JP, Ryu HS. Sonographic findings of placental lacunae and the prediction of adherent placenta in women with placenta previa totalis and prior cesarean section. Ultrasound Obstet Gynecol 2006;28:178-82.

10. Rojas-Losada F, Kuzmar-Daza L, Ferreira-Narváez F, Abdalla M. Diagnóstico y manejo quirúrgico de placenta accreta. Presentación de un caso y revisión de la literatura. Rev Colomb Obstet Ginecol 2006;57:123-8.

11. Guy GP, Peisner DB, Timor-Tritsch IE. Ultrasonographic evaluation of uteroplacental blood flow patterns of abnormally located and adherent placentas. Am J Obstet Gynecol 1990;163:723-7.

12. Hudon L, Belfort M, Broome D. Diagnosis and management of placenta percreta: a review. Obstet Gynecol Surv 1998;53:509-17.

13. Comstock CH. Antenatal diagnosis of placenta accreta: a review. Ultrasound Obstet Gynecol 2005;26:89-96.
14. Chou MM, Ho ES, Lee YH. Prenatal diagnosis of placenta previa accreta by transabdominal color Doppler ultrasound. Ultrasound Obstet Gynecol 2000;15:28-35.

15. Finberg HJ, Williams W. Placenta accreta: prospective sonographic diagnosis in patients with placenta previa and prior cesarean section. J Ultrasound Med 1992;11:333-43.

16. Hoffman-Tretin F, Koenigsberg M, Rabin A, Anyaegbunam A. Placenta accreta. Additional sonographic observations. J Ultrasound Med 1992;11:29-34.

17. Levine D, Hulka CA, Ludmir J, Li W, Edelman RR. Placenta accreta: evaluation with color Doppler US, power Doppler US and MR imaging. Radiology 1997;205:773-6.

18. Perucca E, Cazenave H, Barra A, Ochoa N, Villagrán G, Espinoza R, et al. Placenta previa percreta con invasión vesical. Rev Chil Obstet Ginecol 2002;67:364-7.

19. Thorp JM, Councell RB, Sandridge DA, Wiest HH. Antepartum diagnosis of placenta previa percreta by magnetic resonance imaging. Obstet Gynecol 1992;80:506-8.

20. Catanzarite V, Stanco L, Schrimmer DR, Conroy C. Managing placenta previa/accreta. Contemp Obstet Gynecol 1996;41:66-95. 